\title{
Why is the Sun Flat?
}

OBSERVATIONAL tests of Einstein's general theory of relativity are exceedingly few and far between, and since the twenties observations of the rotation of the orbit of the planet Mercury have provided the most direct support for the theory as a whole. This is why great interest attached to an announcement earlier this year by R. H. Dicke and his colleagues ${ }^{1}$ at Princeton of some measurements of the flatness of the Sun, and to their suggestion that this by itself could account for a part of the rotation of tho orbit of the planet which has so far been attributed to Einstein's theory. If this assertion is true, it is plain that support for the general theory of relativity will be undermined. In the event, however, admiration for the ingenuity and the accuracy of the measurements of the flatness of the Sun has not been accompanied by a general acceptance of Dicke's reasons for believing the Sun should be flattened. It remains to be decided whether the new evidence goes against Einstein's theory.

The facts are these. The orbit of Mercury rotates slowly with respect to the fixed stars chiefly because of the influence of the other planets, but when these: effects have been allowed for there remains a rotation of 43.11 seconds of arc a century to be accounted for. When this was first appreciated at the beginning of the century, a search was made for other explanations than the disturbing influence of the other planets. One possibility then considered was that the Sun might be flattened in such a way as to affect the position of the orbit, although people found it hard to believe that this could happen without more obvious disturbances of the orbit of Mercury than the rotation of the perihelion. In any case, precise measurements of the shape of the Sun were not then practicable. In the event, it may have seemed almost too good to be true that the strictly relativistic calculations of Einstein's theory should imply that there should be a rotation of the orbit of Mercury amounting to 43 seconds of arc a century.

Dicke $^{2}$ was the first to suggest, as early as 1964, that this agreement between experiment and observation might not be as comforting as people had supposed. He pointed out that a flattening of the Sun might well account for a good part of the rotation of the perihelion of Mercury. Dicke and his colleagues have now built and operated an exceedingly clever instrument by means of which light from the outer rim of the Sun is scanned electronically around the circumference in such a way that disturbances due to the Earth's atmosphere can be eliminated. The result, which appears to be beyond dispute, is that the radius of the Sun is greater towards the equator than towards the poles by 34 kilometres, or by roughly five parts in a hundred thousand.

What does this imply for the rotation of the orbit of Mercury? Everything depends on the success with which the gravitational forces of the Sun can be inferred from the shape which is observed. If, of course, the Sun were a mass of fluid held together by its own gravitation, but otherwise at rest, it would be a perfect sphere and it would be possible to infer that the gravitational potential is the same at every point on the surface. (What seems to be the surface would not be a sharp discontinuity between different phases as at the interface between the Earth and its atmosphere, but it would instead be a surface on which the density of solar matter is constant.) The rotation of the Sun as a whole complicates the position, and Dicke says that this accounts for $7 \mathrm{~km}$ of the observed flattening, leaving $27 \mathrm{~km}$ to be accounted for in other ways. For him, this implies that the external gravitation forces of the Sun are not those corrosponding to a perfectly spherical distribution of matter but, rather, to one which is significantly asymmetrical. And if this is indeed the case, some of the obscrvod rotation of the orbit of Mercury $-\mathbf{3 . 4}$ seconds of arc a century - is to be attributed to flattening of the Sun and not to Einstein's theory of relativity.

This is not a big discrepancy. Even if it is safe to assume that the shape of the Sun is a guide to its gravitational influence on the planets, observation and the predictions of theory would differ by only 8 per cent. This, however, is more than the cosmologists could comfortably ignore, and in any case there remains the puzzle of how to account for flattening of the surface. Dicke suggests that it is enough to suppose that the interior of the Sun is rotating a little more quickly than the surface (which makes one revolution in 25.4 days). One immediate difficulty is that any hypothesis like that must somehow be linked with theories of the rotation of the Sun-and in particular with the awkward question of how since the beginning of the Solar System the Sun can have lost enough angular momentum to explain the comparatively slow rotation now observed.

In the circumstances it is not surprising that Dicke's argument is being studied with the greatest care. Professor I. W. Roxburgh ${ }^{3}$ has raised a number of objections, and has, for example, pointed out that if the inside of the Sun were rotating more quickly than the surface, the temperature on the Sun would probably differ by 10 degrees between the equator and the poles. For Roxburgh, temperature gradients within the Sun could account more satisfactorily for the flattening which Dicke has measured and, on that interpretation, observed flattening should have no effect on the gravitational influence of the Sun. Dicke $^{4}$ has refuted some of these arguments, but it would be a great surprise if this important question were now forgotten. In the long run, the controversy could do more to improve understanding of the rotation of the Sun and the structure of its interior than to upset the foundations, such as they are, for the general theory of relativity.

\footnotetext{
' Dicke, R. H., and Goldenberg, H. M., I'hys. Rev. Letters, 18, 313 (1967).

¿ Dicke, R. H., Nature, 202,433 (1964).

'Roxburgh, I. W., Nature, 213, 1077 (1967).

- Dicke, R. H., and Goldenber, H. M., Nature, 214, 1294 (1967).
} 